\title{
Supplement issue for representative papers from the Eighth International Conference on Residual Stresses
}

To increase the service of Powder Diffraction to its readers, ICDD decided in 2007 to publish an additional supplement issue of Powder Diffraction annually in June for representative papers from a powder-diffraction and related conference. The first supplement issue was published in 2008 for articles selected from the Latin American Conference on Applications of Powder Diffraction.

In this 2009 supplement issue, representative papers from the 8th International Conference on Residual Stresses conference (ICRS-8) were selected and edited by I. Cevdet Noyan and Michael B. Prime, and then forwarded to Powder Diffraction for publication. By publishing papers from
ICRS-8, the authors can showcase their experiments and findings to a larger audience, and our readers of Powder Diffraction can learn more about advances in residual stresses from scientists around the world.

Special thanks to our guest editors, Drs. I. Cevdet Noyan and Michael B. Prime, as well as the authors of the representative papers. I also want to acknowledge Nicole Ernst, Denise Flaherty and Carly Bray of ICDD for making sure that everything was in order for this supplement publication.

Ting C. Huang

Editor-in-Chief

\section{The Eighth International Conference on Residual Stresses}

The 8th International Conference on Residual Stresses provided a forum for scientists, academicians, students and engineers interested in the prediction, evaluation, control and application of residual stresses. The aim of this conference was to give equal emphasis to the measurement, modeling and utilization of residual stress/strain data. Both the engineering and scientific aspects of these topics, such as the influence of residual stress fields on distortion, damage initiation, propagation, component lifetimes and failure, were addressed. Special emphasis was given to residual stress aspects of material processing and modeling and measurement on different length scales. Presentations on all stress determination techniques: diffraction (electron, neutron and $\mathrm{x}$-ray), hole-drilling, slitting and contour, indentation, eddy current measurements, Barkhausen noise analysis, acoustic and thermal mapping, etc. were solicited and obtained, as well as papers on the mechanics and modeling of residual stresses. Contributions from industry on residual stress applications and unsolved problems were especially encouraged. All papers submitted to the conference for publication were rigorously reviewed and are available in the conference proceedings disseminated via $\mathrm{CD}$. We have selected sixteen highlights among the submissions for inclusion in this special issue of journal of Powder Diffraction being published to commemorate ICRS-8.

Many papers were selected to describe the state-of-theart diffraction methods currently in use. Advances include measurements at synchrotron sources using very high energy x-ray beams; microbeam diffraction measurements of stress within individual grains of a polycrystalline material, or near device structures in integrated circuits; new laboratory x-ray optics that enable intense parallel beams; application of neutron diffraction to plastic flow in polycrystalline materials and twinning, and practical applications such as stress transfer to reinforcing rods in concrete structures. While these techniques are very popular, and becoming more so, they are not universally applicable, and data acquisition, analysis and interpretation requires care.

Several papers were selected to illustrate some important and not fully resolved issues in residual stress. Some papers show that no one technique alone can measure everything that is needed to evaluate the structural integrity of industrial components. Destructive measurements and other nondiffraction measurements play a crucial and complementary role. Other papers deal with some of the challenges involved in taking measurements on test coupons and then using the results on full-scale structures. Some papers illustrate another important statistical issue. For industrialists to implement and take credit for life extension treatments that involve compressive residual stresses, two things are necessary: (1) a reliable and predictable process and (2) a reliable and repeatable measurement method in order to verify (Bunch, J. O., Private Communication). It can be very hard to sort out how much each issue contributes to the inevitable variability in results.

Since there are many techniques that can be used to measure residual stress, choosing a technique for a specific application requires care. In fact, the information volume- the volume to which the measurement values are referred tocan be quite different when different techniques are applied to the same specimen. Understanding the intended application of the results is also critical in selecting a technique. Measuring stresses to predict part distortion after machining, or during fatigue crack growth of macroscopic cracks, or in the microscopic initiation phase of intergranular stress corrosion cracks, would require different capabilities from the measurement technique. We exhort the practitioners to use multiple techniques whenever possible. Different techniques often use drastically different underlying assumptions. Getting diverse techniques to agree, or understanding the differences if they do not, is often the only way to get a true 
understanding of the stress state. All data should be reported with the proper error bounds. Compatibility between modeling and measurement should be ensured with the same set of assumptions used in both. Meticulous care in measurement planning, data acquisition, and data analysis and error propagation, rigorously documented will ensure that residual stress analysis will be a valuable contribution to engineering design and manufacturing.

Parochialism and isolated research will not solve the fascinating scientific and technical challenges of understanding and manipulating residual stresses and their effects. There is no foreseeable technology that will allow us to measure the full three-dimensional distribution of all six components of the stress tensor in real parts of arbitrary dimensions. In addition, because of the complicated thermo-mechanical processes involved, it will not be possible to accurately predict the stresses. Nor will it be possible to predict all failures with deterministic models. Insightful use of multiple measurement techniques, numerical modeling, and stochastic understand- ing is clearly needed. We need hybrid approaches that start with our limited data, apply physical constraints and process knowledge, and thereby expand the results to cover the regions and regimes of relevance. Only with such a comprehensive approach can we realize the full potential of science and technology to understand, predict, and manipulate stresses that affect the manufacturability, cost, performance, and safety of the systems that we enjoy and rely upon in our daily lives. We hope that the meeting-of-minds at the ICRS-8 conference, and this special publication summarizing the highlights, will contribute to this endeavor.

Bunch, J. O., Private Communication, Boeing Company.

\section{Cevdet Noyan Columbia University}

Michael B. Prime Los Alamos National Laboratory 